Mathematical Research Letters 6, 345-356 (1999)

\title{
FIELDS GALOIS-EQUIVALENT TO A LOCAL FIELD OF POSITIVE CHARACTERISTIC
}

\author{
IDO EFRAT AND IVAN FESENKO
}

\section{Introduction}

A celebrated theorem of Artin and Schreier [AS] characterizes the fields $K$ whose absolute Galois group $G_{K}$ is isomorphic to that of $\mathbb{R}$ as the real closed fields. In the present paper we consider the analogous problem for nonarchimedean local fields of positive characteristic $F=\mathbb{F}_{p^{n}}((t))$. We show that a field $K$ with absolute Galois group isomorphic to $G_{F}$ possesses a Henselian valuation $v$ such that:

(1) the value group $\Gamma$ of $v$ satisfies $\Gamma / l \cong \mathbb{Z} / l$ for all prime numbers $l \neq p$;

(2) the residue field $\bar{K}$ of $v$ has characteristic $p$;

(3) the maximal prime to $p$ Galois group $G_{\bar{K}}\left(p^{\prime}\right)$ of $\bar{K}$ is $\hat{\mathbb{Z}} / \mathbb{Z}_{p}$;

(4) if char $K=0$ then $\Gamma=p \Gamma$ and $\bar{K}$ is perfect.

For every positive integer $r$ we construct such fields $K$ of characteristic $p$ with $\Gamma / p \cong(\mathbb{Z} / p)^{r}$. Likewise we construct examples with $\Gamma \cong \mathbb{Z}, G_{\bar{K}} ¥ \hat{\mathbb{Z}}$ and $\bar{K}$ imperfect.

The similar problem for $p$-adic fields was answered by Koenigsmann [Kn] and the first named author [E1] (for $p \neq 2$ ), extending earlier results by Neukirch [N2] and Pop [P1]: the fields $K$ such that $G_{K} \cong G_{F}$ for some finite extension $F$ of $\mathbb{Q}_{p}$ are precisely the $p$-adically closed fields in the sense of [PR].

\section{Notation}

We denote the algebraic, separable, and inseparable closures of a field $K$ by $\tilde{K}, K_{\text {sep }}$, and $K_{\text {ins }}$, respectively. For a positive integer $m$ with char $K \nmid m$ let $\mu_{m}$ be the group of roots of unity of order dividing $m$ in $\tilde{K}$. For a prime $l$ with $l \neq \operatorname{char} K$ let $\mu_{l^{\infty}}=\lim _{\rightarrow} \mu_{l^{r}}$. Given a profinite group $G$ and a prime number $l$, denote the quotient $\underset{\leftarrow}{\longrightarrow} G / N$, where $N$ ranges over all open normal subgroups of $G$ with $G / N$ abelian (resp., of $l$-power order, of order prime to $l$ ) by $G($ ab) (resp., $\left.G(l), G\left(l^{\prime}\right)\right)$. We define $G(\mathrm{ab}, l), G\left(\mathrm{ab}, l^{\prime}\right)$ similarly. For a (Krull) valuation $v$ on $K$ let $\Gamma_{v}, O_{v}$, and $\bar{K}_{v}$ be the corresponding value group, valuation ring, and residue field, respectively.

Received February 11, 1999.

Research supported by EPSRC grant GR/M08356.

1991 Mathematics Subject Classification: primary 11S20, secondary 12J10 


\section{Galois groups of Henselian fields}

We first recall several basic facts about the structure of the decomposition group of $(K, v)$ relative to $K_{\text {sep }}$ (see e.g. [Ed], [P2, 1$]$ or [E1, 11$]$ for more details and proofs). For simplicity we assume here that $v$ is Henselian, i.e., the decomposition group is $G_{K}$. Let $K_{\mathrm{ur}}$ and $K_{\mathrm{tr}}$ be the maximal unramified and maximal tamely ramified Galois extensions of $(K, v)$, respectively. If $p=$ char $\bar{K}_{v}>0$ then $G_{K_{\mathrm{tr}}}$ is the unique $p$-Sylow subgroup of $G_{K_{\mathrm{ur}}}$. If char $\bar{K}_{v}=0$ then $G_{K_{\mathrm{tr}}}=1$. There are natural short exact sequences

$$
\begin{gathered}
1 \rightarrow \operatorname{Gal}\left(K_{\mathrm{tr}} / K_{\mathrm{ur}}\right) \rightarrow \operatorname{Gal}\left(K_{\mathrm{tr}} / K\right) \rightarrow G_{\bar{K}_{v}} \rightarrow 1 \\
1 \rightarrow G_{K_{\mathrm{tr}}} \rightarrow G_{K} \rightarrow \operatorname{Gal}\left(K_{\mathrm{tr}} / K\right) \rightarrow 1
\end{gathered}
$$

which are split, by $[\mathrm{N} 1]$ and $[\mathrm{KPR}]$, respectively. For a prime number $l$ let $\delta_{l}=\operatorname{dim}_{\mathbb{F}_{l}} \Gamma_{v} / l$. Then $\operatorname{Gal}\left(K_{\mathrm{tr}} / K_{\mathrm{ur}}\right) \cong \prod_{l \neq \operatorname{char} \bar{K}_{v}}\left(\lim _{r} \mu_{l^{r}}\right)^{\delta_{l}}$ as $G_{\bar{K}_{v}}$-modules. In particular, if $\Gamma_{v} \cong \mathbb{Z}$ and $\operatorname{char} \bar{K}_{v}=p>0$ then $\delta_{l}=1$ for all primes $l$, so the $G_{\bar{K}_{v}}$-module $\operatorname{Gal}\left(K_{\text {tr }} / K_{\text {ur }}\right)$ is $\hat{\mu}=\lim _{(m, p)=1} \mu_{m}$, and $\operatorname{Gal}\left(K_{\text {tr }} / K\right) \cong \hat{\mu} \rtimes G_{\bar{K}_{v}}$ with the Galois action.

The analogous result for the maximal pro- $l$ Galois group $G_{K}(l)$ of $K$ is the following: If $l \neq \operatorname{char} \bar{K}_{v}$ is prime and $\mu_{l} \subseteq K$ then $G_{K}(l) \cong \mathbb{Z}_{l}^{\delta_{l}} \rtimes G_{\bar{K}_{v}}(l)$, where $\sigma \in G_{\bar{K}_{v}}(l)$ acts on $\tau \in \mathbb{Z}_{l}^{\delta_{l}}$ according to $\sigma \tau \sigma^{-1}=\chi_{\bar{K}_{v}, l}(\sigma) \tau$; here $\chi_{\bar{K}_{v}, l}: G_{\bar{K}_{v}}(l) \rightarrow 1+l \mathbb{Z}_{l}$ is the pro-l cyclotomic character of $\bar{K}_{v}$, induced by the restriction homomorphism $G_{\bar{K}_{v}}(l) \rightarrow \operatorname{Aut}\left(\mu_{l^{\infty}}\right) \cong \mathbb{Z}_{l}^{\times}$.

Now fix a prime number $p$. Given a pro- $p$ group $H$ and a cardinal number $c$ let $F_{p}(H ; c)$ be the free $H$-operator pro- $p$ group on $c$ generators, in the sense of $[\mathrm{K} 1],[\mathrm{MSh}]$. The following is a modest generalization of the main result of [MSh] (which treats Laurent series fields).

Theorem 1.1. Let $(K, v)$ be a Henselian discretely valued field of characteristic $p$ and let $c=\max \left\{\aleph_{0},\left|\bar{K}_{v}\right|\right\}$. Then $G_{K_{\mathrm{tr}}} \cong F_{p}\left(\operatorname{Gal}\left(K_{\mathrm{tr}} / K\right) ; c\right)$ as $\operatorname{Gal}\left(K_{\mathrm{tr}} / K\right)$ operator pro-p groups; in particular, $G_{K} \cong F_{p}\left(\operatorname{Gal}\left(K_{\mathrm{tr}} / K\right) ; c\right) \times \operatorname{Gal}\left(K_{\mathrm{tr}} / K\right)$ with the canonical action.

When $k=\bar{K}_{v}$ is perfect, this theorem can be proven using precisely the same argument as in [MSh, Th. 1]. We therefore omit the details. When $k$ is not perfect, one can prove it as follows: Let $u$ be the unique prolongation of $v$ to $L=k_{\text {ins }} K$. The restriction $G_{L} \rightarrow G_{K}$ is an isomorphism mapping $G_{L_{\mathrm{ur}}}$, $G_{L_{\mathrm{tr}}}$ onto $G_{K_{\mathrm{ur}}}, G_{K_{\mathrm{tr}}}$, respectively. By the result for perfect residue fields, $G_{L_{\mathrm{tr}}} \cong F_{p}\left(\operatorname{Gal}\left(L_{\mathrm{tr}} / L\right) ; c\right)$ as $\operatorname{Gal}\left(L_{\mathrm{tr}} / L\right)$-operator groups. It follows that $G_{K_{\mathrm{tr}}} \cong$ $F_{p}\left(\operatorname{Gal}\left(K_{\mathrm{tr}} / K\right) ; c\right)$ as $\mathrm{Gal}\left(K_{\mathrm{tr}} / K\right)$-operator groups, as desired.

Corollary 1.2. Let $\left(K_{1}, v_{1}\right),\left(K_{2}, v_{2}\right)$ be Henselian discretely valued fields of characteristic $p$, and let $\bar{K}_{1}, \bar{K}_{2}$ be the corresponding residue fields. Suppose that 
$G_{\bar{K}_{1}} \cong G_{\bar{K}_{2}}$, that this isomorphism is compatible with the Galois actions on the roots of unity, and that $\max \left\{\aleph_{0},\left|\bar{K}_{1}\right|\right\}=\max \left\{\aleph_{0},\left|\bar{K}_{2}\right|\right\}$. Then $G_{K_{1}} \cong G_{K_{2}}$.

Proof. We have $\operatorname{Gal}\left(K_{1, \operatorname{tr}} / K_{1}\right) \cong \hat{\mu} \rtimes G_{\bar{K}_{1}} \cong \hat{\mu} \rtimes G_{\bar{K}_{2}} \cong \operatorname{Gal}\left(K_{2, \operatorname{tr}} / K_{2}\right)$ with the Galois actions. Now apply Theorem 1.1.

Proposition 1.3. Let $(K, v)$ be a Henselian discretely valued field of characteristic p. Suppose that $|K|=\max \left\{\aleph_{0},\left|\bar{K}_{v}\right|\right\}$. Let $L$ be a maximal totally tamely ramified extension of $(K, v)$. Let $(E, u)$ be a Henselian discretely valued field of characteristic $p$ with $\bar{E}_{u}=L$. Then $G_{E} \cong G_{K}$.

Proof. Let $c=|K|=\max \left\{\aleph_{0},\left|\bar{K}_{v}\right|\right\}$, let $H=\hat{\mu} \rtimes G_{\bar{K}_{v}}$ with the Galois action, and let $V=F_{p}(H ; c)$. By Theorem 1.1, $V \cong G_{K_{\mathrm{tr}}}$ and $G_{K} \cong V \rtimes H$. Since $\tilde{\mathbb{F}}_{p} \subseteq K_{\mathrm{tr}}$, the Galois action of $V$ on $\hat{\mu}$ is trivial. Further, the unique prolongation of $v$ to $L$ has residue field $\bar{K}_{v}$. Hence $G_{L} \cong V \rtimes G_{\bar{K}_{v}}$, and this isomorphism is compatible with the Galois action on $\hat{\mu}$. Therefore

$$
\operatorname{Gal}\left(E_{\mathrm{tr}} / E\right) \cong \hat{\mu} \rtimes G_{L} \cong \hat{\mu} \times\left(V \rtimes G_{\bar{K}_{v}}\right) \cong V \rtimes\left(\hat{\mu} \rtimes G_{\bar{K}_{v}}\right)=V \rtimes H .
$$

Now from [MSh, $\S 1$, Prop. 1] we deduce that

$$
\begin{aligned}
F_{p}(V \rtimes H ; c) \rtimes(V \rtimes H) & \cong\left(F_{p}(H ; c) * V\right) \rtimes H \\
& =\left(F_{p}(H ; c) * F_{p}(H ; c)\right) \rtimes H \cong F_{p}(H ; c) \rtimes H=V \rtimes H,
\end{aligned}
$$

where $*$ denotes free pro- $p$ product. Since $|L|=|K|$, from Theorem 1.1 we deduce

$$
\begin{aligned}
G_{E} \cong F_{p}\left(\operatorname{Gal}\left(E_{\mathrm{tr}} / E\right) ; c\right) \rtimes \operatorname{Gal}\left(E_{\mathrm{tr}} / E\right) \cong \\
\qquad F_{p}(V \rtimes H ; c) \rtimes(V \rtimes H) \cong V \rtimes H \cong G_{K} .
\end{aligned}
$$

\section{Existence of Henselian valuations}

Let $K_{2}^{M}(E)$ be the second Milnor $K$-group of the field $E$, and let $\{\cdot, \cdot\}: E^{\times} \times$ $E^{\times} \rightarrow K_{2}^{M}(E)$ be the natural symbolic map. The following theorem combines powerful constructions of Ware [Wr], Arason-Elman-Jacob [AEJ] (for $l=2$ ), and Hwang-Jacob [HJ] (for $l \neq 2$ ); see also [E3] and [Kn].

Theorem 2.1. Let $l$ be a prime number, let $E$ be a field of characteristic $\neq l$, let $T$ be a subgroup of $E^{\times}$containing $\left(E^{\times}\right)^{l}$ and -1 . Suppose that:

(i) For every $x, y \in E^{\times}$which are $\mathbb{F}_{l}$-linearly independent in $E^{\times} / T$ one has $\{x, y\} \neq 0$ in $K_{2}^{M}(E)$

(ii) For every $x \in E^{\times} \backslash T$ and $y \in T \backslash\left(E^{\times}\right)^{l}$ one has $\{x, y\} \neq 0$ in $K_{2}^{M}(E)$. Then there exists a valuation $u$ on $E$ such that $\left(\Gamma_{u}: l \Gamma_{u}\right) \geq\left(E^{\times}: T\right) / l$ and $u(l) \neq 0$. Furthermore, if $\bar{E}_{u}=\bar{E}_{u}^{l}$ then $\left(\Gamma_{u}: l \Gamma_{u}\right) \geq\left(E^{\times}: T\right)$.

The rank of a profinite group is the minimal number (possibly $\infty$ ) of topological generators of it. 
Proposition 2.2. [E1, Prop. 2.1] Let $l$ be a prime number and let $(E, u)$ be a valued field such that char $\bar{E}_{u} \neq l$ and $G_{\bar{E}_{u}}(l)$ is infinite. Suppose that

$$
\sup _{M} \operatorname{rank} G_{M}(l)<\infty
$$

where $M$ ranges over all finite separable extensions of $E$. Then $(E, u)$ is Henselian.

Combining the previous two facts, we obtain the following result (which is essentially proven in [E1] for $l=2$ ).

Proposition 2.3. Let $l, p$ be distinct prime numbers and let $K$ be a field of characteristic $\neq l$. Let $E_{0}$ be a finite extension of $K$ containing $\mu_{l}$ and containing $\sqrt{-1}$ if $l=2$. Suppose that for every finite separable extension $E$ of $E_{0}$ one has

$$
G_{E}(l) \cong\left\langle\sigma, \tau \mid \sigma \tau \sigma^{-1}=\tau^{p^{s}}\right\rangle_{\mathrm{pro}-l}
$$

for some $s=s(E) \geq 1$ such that $p^{s} \equiv 1 \bmod l$. Then there exists a Henselian valuation $v$ on $K$ such that $\Gamma_{v} / l \cong \mathbb{Z} / l$, char $\bar{K}_{v} \neq l$, and $\bar{K}_{v}$ is not algebraically closed.

Proof. For $E$ as above denote $H^{i}(E)=H^{i}\left(G_{E}(l), \mathbb{Z} / l\right)$. We consider the cup product $H^{1}(E) \times H^{1}(E) \rightarrow H^{2}(E)$. Let $\varphi_{1}, \varphi_{2}$ be an $\mathbb{F}_{l}$-linear basis of $H^{1}(E)$ which is dual to the basis of $G_{E}(l) / G_{E}(l)^{l}\left[G_{E}(l), G_{E}(l)\right]$ consisting of the images of $\sigma$ and $\tau$. From the defining relation $\tau^{p^{s}-1}[\tau, \sigma]=1$ of $G_{E}(l)$ we deduce that $\varphi_{1} \cup \varphi_{2} \neq 0[\mathrm{~K} 2, \S 7.8]$. Furthermore, when $l \neq 2$ one has $\varphi_{1} \cup \varphi_{1}=\varphi_{2} \cup \varphi_{2}=0$ by the anti-commutativity of $\cup$. When $l=2$ we may identify $\varphi_{i} \cup \varphi_{i}$ with the class of a quaternion algebra $\left(a_{i}, a_{i} / E\right)$ in the Brauer group $\operatorname{Br}(E)$; here $a_{i}\left(E^{\times}\right)^{2}$ corresponds to $\varphi_{i}$ under the Kummer isomorphism $E^{\times} /\left(E^{\times}\right)^{2} \cong H^{1}(E)$. Since $\left(a_{i}, a_{i} / E\right)=\left(a_{i},-1 / E\right)$ in $\operatorname{Br}(E)$ and $\sqrt{-1} \in E$ we obtain that $\varphi_{i} \cup \varphi_{i}=0$, $i=1,2$, in this case as well. Consequently, $H^{2}(E) \cong \wedge^{2} H^{1}(E)$.

By the Kummer theory and the Merkur'ev-Suslin theorem [MSu], this implies that $K_{2}^{M}(E) / l \cong \wedge^{2}\left(E^{\times} / l\right)$ naturally. Hence (i) and (ii) of Theorem 2.1 hold for $T=\left(E^{\times}\right)^{l}$. Since $\operatorname{dim}_{\mathbb{F}_{l}}\left(E^{\times} /\left(E^{\times}\right)^{l}\right)=\operatorname{rank} G_{E}(l)=2$, Theorem 2.1 therefore gives rise to a valuation $u$ on $E$ such that $\operatorname{dim}_{\mathbb{F}_{l}}\left(\Gamma_{u} / l\right) \geq 1$ and char $\bar{E}_{u} \neq l$.

Furthermore, if $\bar{E}_{u}=\bar{E}_{u}^{l}$ then $\delta_{l}=\operatorname{dim}_{\mathbb{F}_{l}}\left(\Gamma_{u} / l\right) \geq 2$ by the last statement of Theorem 2.1. From the discussion in $\S 1$ this would imply $\mathbb{Z}_{l}^{2} \leq G_{E}(l)$, which is not the case [E1, Lemma 4.1]. We conclude that $G_{\bar{E}_{u}}(l) \neq 1$. This implies that the latter group is in fact infinite ([B]; note that when $l=2, \sqrt{-1} \in \bar{E}_{u}$ ). Proposition 2.2 therefore shows that $(E, u)$ is Henselian.

Now take $E=E_{0}$, let $u$ be as above, and let $v=\operatorname{Res}_{K} u$. Then $\operatorname{dim}_{\mathbb{F}_{l}}\left(\Gamma_{v} / l\right)=$ $\operatorname{dim}_{\mathbb{F}_{l}}\left(\Gamma_{u} / l\right) \geq 1\left[\mathrm{E} 1\right.$, Lemma 1.2] and char $\bar{K}_{v} \neq l$. Since the finite extension $\bar{E}_{u}$ of $\bar{K}_{v}$ is not algebraically closed, neither is $\bar{K}_{v}$. Also, Henselianity goes down in finite extensions, provided that the upper residue field is not separably closed [Eg, Cor. 3.5]. Therefore $(K, v)$ is Henselian. 
Valuations $v, v^{\prime}$ are called comparable if one of $O_{v}, O_{v^{\prime}}$ contains the other.

Proposition 2.4. (Endler-Engler [EE, Prop.]) Let $v, v^{\prime}$ be valuations on a field $K$. Suppose that $v$ is Henselian and that $\bar{K}_{v^{\prime}}$ is not algebraically closed. Then $v, v^{\prime}$ are comparable.

Lemma 2.5. Let $(L, w) /(K, v)$ be a Galois extension of Henselian valued fields of degree $n$. Suppose that the norm homomorphism $N_{L / K}: L^{\times} \rightarrow K^{\times}$is surjective. Then $\left(\Gamma_{v}: n \Gamma_{v}\right)=\left(\Gamma_{w}: \Gamma_{v}\right)$.

Proof. By the Henselianity, one has a well-defined commutative square

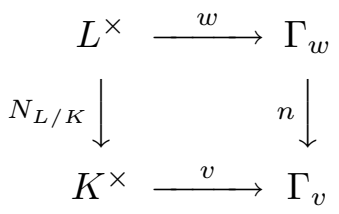

By assumption, the left vertical map is surjective. Hence so is the right vertical map; i.e., $\Gamma_{v}=n \Gamma_{w}$. By [E1, Lemma 1.2] again,

$$
\left(\Gamma_{v}: n \Gamma_{v}\right)=\left(\Gamma_{w}: n \Gamma_{w}\right)=\left(\Gamma_{w}: \Gamma_{v}\right) .
$$

\section{The absolute Galois group of a local field of characteristic $p$}

From now on we fix a local field $F=\mathbb{F}_{q}((t))$ of characteristic $p>0$. Let $G=G_{F}, T=G_{F_{\mathrm{ur}}}$, and $V=G_{F_{\mathrm{tr}}}$, taken with respect to the canonical discrete valuation on $F$. The group structure of $G$ was described by Koch [K1] (and follows from the general considerations in $\S 1)$; namely:

(i) $G=V \times(G / V)$;

(ii) $T / V \cong \hat{\mathbb{Z}} / \mathbb{Z}_{p}$;

(iii) $G / T \cong \hat{\mathbb{Z}}$;

(iv) $G / V \cong(T / V) \rtimes(G / T)$, where a generator $\sigma$ of $G / T$ acts on a generator $\tau$ of $T / V$ according to the Hasse-Iwasawa relation $\sigma \tau \sigma^{-1}=\tau^{q}$;

(v) $V \cong F_{p}\left(G / V ; \aleph_{0}\right)$; in particular, $V$ is a free pro- $p$ group on countably many generators [MSh, $\S 1$, Lemma 4].

\section{Proposition 3.1.}

(a) $T / V$ intersects non-trivially every non-trivial normal closed subgroup of $G / V$.

(b) $T$ intersects non-trivially every non-trivial normal closed subgroup of $G$.

Proof. (a) We need to show that if $L$ is a Galois extension of $F$ such that $L F_{\mathrm{ur}}=F_{\mathrm{tr}}$ then $L=F_{\mathrm{tr}}$. To this end, denote $L^{\prime}=L \cap F_{\mathrm{ur}}$. Then $\operatorname{Gal}\left(F_{\mathrm{tr}} / L^{\prime}\right) \cong$ $\operatorname{Gal}\left(F_{\mathrm{tr}} / F_{\mathrm{ur}}\right) \times \operatorname{Gal}\left(F_{\mathrm{ur}} / L^{\prime}\right)$. In particular, $\operatorname{Gal}\left(F_{\mathrm{tr}} / L^{\prime}\right)$ is abelian, by (ii) and (iii) above. For each positive integer $m$ which is prime to $p$ choose $t_{m} \in F_{\text {tr }}$ such that $t_{m}^{m}=t$. The abelianity implies that $L^{\prime}\left(t_{m}\right) / L^{\prime}$ is normal. Since $L^{\prime} / F$ is unramified it follows that $\mu_{m} \subseteq L^{\prime}$. Conclude that $F_{\text {ur }}=\bigcup_{(m, p)=1} F\left(\mu_{m}\right) \subseteq L^{\prime}$, whence $L=L F_{\text {ur }}=F_{\mathrm{tr}}$.

(b) follows from (a). 
Lemma 3.2. Let $E$ be a totally ramified extension of $F$ of prime degree $l$ and let $\sigma$ be a generator of $\operatorname{Gal}(E / F)$. Let $v$ be the canonical valuation on $E$ and let $\pi$ be a prime element of $E$. Let $s$ be the maximal integer such that the sth ramification group of $\operatorname{Gal}(E / F)$ is non-trivial. Then:

(a) $v\left((\sigma-1)\left(\pi^{n}\right)\right)=s+n$ for every integer $n$ relatively prime to $p l$;

(b) $E /(F+\wp(E))$ is infinite.

Proof. (a) When $l=p$ this is proven in [FV, Ch. III, (1.4)]. Suppose $l \neq p$. Then $s=0[\mathrm{FV}$, Ch. II, $\S 4.4$, Cor. 1] and $\sigma(\pi)=\zeta \pi$ for a primitive $l$ th root of unity $\zeta$. Hence $(\sigma-1)\left(\pi^{n}\right)=\left(\zeta^{n}-1\right) \pi^{n}$. It remains to observe that $v\left(\zeta^{n}-1\right)=0$.

(b) In all cases except $l=p=2$ let $I$ be the set of all integers $n$ such that $n<-s$ and $(p l, n(s+n))=1$. When $l=p=2$ let $I$ be the set of all integers $n$ such that $2 \nmid n$ and $4 \nmid s+n<0$. Using again [FV, Ch. II, §4.4, Cor. 1] we see that $I$ is always infinite.

We claim that the elements $\pi^{n}$, where $n \in I$, are distinct modulo $F+\wp(E)$. Indeed, suppose that $\pi^{n}-\pi^{n^{\prime}}=y+\wp(x)$, with $y \in F, x \in E, n, n^{\prime} \in I$, and $n<n^{\prime}$. By (a),

$$
0>s+n=v\left((\sigma-1)\left(\pi^{n}-\pi^{n^{\prime}}\right)\right)=v(\wp((\sigma-1)(x))) .
$$

However, negative elements of $v(\wp(E))$ are divisible by $p$. Thus, we get a contradiction in all cases except $l=p=2$.

In the remaining case $l=p=2$ we obtain $v(x)<0$ and hence $v((\sigma-$ $1)(\wp(x)))=2 v((\sigma-1)(x))$. Since $\pi$ is a primitive element for the extension $E / F$, we can write $x=c_{0}+c_{1} \pi$ with $c_{0}, c_{1} \in F$. Then

$$
v((\sigma-1)(x))=v\left(c_{1}\right)+v((\sigma-1)(\pi))=v\left(c_{1}\right)+s+1,
$$

by (a). But $2 \mid v\left(c_{1}\right)$ and $2 \nmid s$ [FV, Ch. III, Prop. 2.3], so $v((\sigma-1)(x))$ is even. We conclude that $4 \mid s+n$, a contradiction.

Proposition 3.3. $V$ intersects non-trivially every non-trivial normal closed subgroup of $G$.

Proof. (Compare [P1, Satz 1.4].) Let $H$ be a non-trivial normal closed subgroup of $G$ and let $L$ be its fixed field. It follows from Proposition 3.1(b) that $L F_{\text {ur }} \neq$ $F_{\text {sep }}$. Hence we can take a finite Galois extension $N$ of $F$ such that $N \nsubseteq L F_{\text {ur }}$. Denote the maximal elementary $p$-abelian Galois extension of $N$ by $N[p]$. It is a Galois extension of $F$. Set $K=L \cap N$ and $M=L \cap N[p]$. Then $N \nsubseteq K K F_{\text {ur }}$, i.e., the extension $N / K$ has a non-trivial inertia group. Since $\operatorname{Gal}(N / K)$ is solvable, we may therefore find an intermediate field $K \subseteq N_{0} \subset N$ such that $N / N_{0}$ is a totally ramified extension of prime degree. By Lemma 3.2(b), $N /\left(N_{0}+\wp(N)\right)$ is infinite. Hence so is $N /(K+\wp(N))$.

By the Artin-Schreier theory, the dual of the natural homomorphism $K / \wp(K) \rightarrow N / \wp(N)$ may be canonically identified with the restriction homomorphism $\operatorname{Gal}(N[p] / N) \rightarrow \operatorname{Gal}(K[p] / K)$. Since the cokernel $N /(K+\wp(N))$ of 
the former homomorphism is infinite, so is the kernel $\operatorname{Gal}(N[p] / K[p] N)$ of the latter homomorphism.

Now the group $\operatorname{Gal}(M / K) \cong \operatorname{Gal}(M N / N)$ is an epimorphic image of $\operatorname{Gal}(N[p] / N)$, hence $M \subseteq K[p]$. It follows that $\operatorname{Gal}(N[p] / M N)$ is infinite. Since it is an elementary abelian $p$-group, it is not cyclic. Therefore the $p$-Sylow subgroups of $\operatorname{Gal}(L N[p] / L) \cong \operatorname{Gal}(N[p] / M)$ are not cyclic (note that as $N[p] / F$ is Galois, so are $L N[p] / L$ and $N[p] / M)$. It follows that $\operatorname{Syl}_{p}\left(G_{L}\right)$ is not cyclic. On the other hand, $G_{L} /\left(G_{L} \cap V\right)$ embeds in $G / V \cong\left(\hat{\mathbb{Z}} / \mathbb{Z}_{p}\right) \times \hat{\mathbb{Z}}$, hence its $p$-Sylow subgroups are cyclic. Conclude that $H \cap V=G_{L} \cap V \neq 1$, as required.

\section{The main results}

We still fix a local field $F=\mathbb{F}_{q}((t))$ of characteristic $p>0$.

Theorem 4.1. Let $K$ be a field with $G_{K} \cong G_{F}$. There exists a Henselian valuation $v$ on $K$ such that:

(a) $\left(\Gamma_{v}: l \Gamma_{v}\right)=l$ for all primes $l \neq p$;

(b) $\operatorname{char} \bar{K}_{v}=p$.

Proof. Fix an isomorphism $\sigma: G_{K} \rightarrow G_{F}$. For a separable extension $E$ of $K$ let $E^{\prime}$ denote the separable extension of $F$ such that $\sigma G_{E}=G_{E^{\prime}}$.

Let $l \neq p$ be a prime number. Then $\operatorname{cd}_{l}\left(G_{K}\right)=\operatorname{cd}_{l}\left(G_{F}\right)=2$ [S, II-15, Prop. $12]$, so char $K \neq l$ [S, II-4, Prop. 3]. Fix a finite separable extension $E_{l}$ of $K$ such that $E_{l}, E_{l}^{\prime}$ contain $\mu_{l}$, and contain $\sqrt{-1}$ if $l=2$. Then for every finite separable extension $E$ of $E_{l}$ one has

$$
G_{E}(l) \cong G_{E^{\prime}}(l) \cong\left\langle\sigma, \tau \mid \sigma \tau \sigma^{-1}=\tau^{p^{s}}\right\rangle_{\text {pro }-l}
$$

for some $s=s(E) \geq 1$ such that $p^{s} \equiv 1 \bmod l$ (namely, $p^{s}$ is the cardinality of the residue field of $E^{\prime}$; see $\S 1$ ). Proposition 2.3 gives rise to a Henselian valuation $v_{l}$ on $K$ such that $\Gamma_{v_{l}} / l \cong \mathbb{Z} / l$, char $\bar{K}_{v_{l}} \neq l$, and $\bar{K}_{v_{l}}$ is not algebraically closed.

By Proposition 2.4, the valuations $v_{l}, l \neq p$, are pairwise comparable. It follows that $\bigcap_{l \neq p} O_{v_{l}}$ is a Henselian valuation ring on $K$. Let $v$ be the corresponding valuation on $K$. For every prime number $l \neq p$ the fact that $O_{v} \subseteq O_{v_{l}}$ implies that $\Gamma_{v_{l}}$ is an epimorphic image of $\Gamma_{v}$; hence $\operatorname{dim}_{\mathbb{F}_{l}}\left(\Gamma_{v} / l\right) \geq \operatorname{dim}_{\mathbb{F}_{l}}\left(\Gamma_{v_{l}} / l\right)=1$. Moreover, $\mathbb{Z}_{l}^{2} \not \leq G_{E_{l}}(l)$ [E1, Lemma 4.1]. We conclude as before using [E1, Lemma 1.2] and the considerations of $\S 1$ that $\operatorname{dim}_{\mathbb{F}_{l}}\left(\Gamma_{v} / l\right)=1$, proving (a).

To prove (b), let $T_{v}, V_{v}$ be the inertia and ramification groups, respectively, of $v$ in $K_{\mathrm{sep}} / K$. For every prime number $l \neq p$, char $\bar{K}_{v}$, part (a) gives $\operatorname{Syl}_{l}\left(T_{v} / V_{v}\right) \cong \mathbb{Z}_{l}$. In particular, $T_{v} / V_{v}$ is non-trivial. Now the closed normal subgroup $\sigma^{-1}(V)$ of $G_{K}$ is free pro- $p$ of infinite rank. According to Proposition 3.3 it intersects every non-trivial closed normal subgroup of $G_{K}$. Thus $T_{v} \cap \sigma^{-1}(V) \neq 1$. Since a free pro- $p$ group of rank $\geq 2$ does not have non-trivial abelian closed normal subgroups, $T_{v} \cap \sigma^{-1}(V)$ is non-abelian. Therefore $\operatorname{Syl}_{p}\left(T_{v}\right)$ is non-abelian, which can happen only when char $\bar{K}_{v}=p$. 
Lemma 4.2. Let $H$ be a profinite group such that $\operatorname{cd}_{p}(H) \leq 1$ and such that $\operatorname{Syl}_{l}(H) \cong \mathbb{Z}_{l}$ for all primes $l \neq p$. Then $H\left(p^{\prime}\right) \cong \hat{\mathbb{Z}} / \mathbb{Z}_{p}$.

Proof. By [S, I-23, Prop. 16] and [FJ, Cor. 20.14], $H$ embeds as a closed subgroup of a free profinite group $\hat{F}$. Now any closed subgroup of $\hat{F}$ isomorphic to $\mathbb{Z}_{l}$, $l \neq p$, is mapped bijectively by the canonical projection $\hat{F} \rightarrow \hat{F}\left(\mathrm{ab}, p^{\prime}\right)$. Since the induced homomorphism $H \rightarrow \hat{F}\left(\mathrm{ab}, p^{\prime}\right)$ breaks through $H\left(\mathrm{ab}, p^{\prime}\right)$, any $l$-Sylow subgroup of $H$ is mapped bijectively onto an $l$-Sylow subgroup of $H\left(\mathrm{ab}, p^{\prime}\right)$. It follows that $H\left(\mathrm{ab}, p^{\prime}\right) \cong \prod_{l \neq p} \mathbb{Z}_{l} \cong \hat{\mathbb{Z}} / \mathbb{Z}_{p}$. Since $\operatorname{cd}\left(\hat{\mathbb{Z}} / \mathbb{Z}_{p}\right) \leq 1$, the projection $H\left(p^{\prime}\right) \rightarrow H\left(\mathrm{ab}, p^{\prime}\right)$ has a continuous homomorphic section. Then $H\left(p^{\prime}\right)$ and the image of this section have the same $l$-Sylow subgroups, hence they coincide. Thus $H\left(p^{\prime}\right) \cong \hat{\mathbb{Z}} / \mathbb{Z}_{p}$.

Proposition 4.3. Let $K$ and $v$ be as in Theorem 4.1 and let $l \neq p$ be a prime number. Then:

(a) $G_{\bar{K}_{v}}\left(p^{\prime}\right) \cong \hat{\mathbb{Z}} / \mathbb{Z}_{p}$.

(b) For $s \geq 0, \mu_{l^{s}} \subseteq \bar{K}_{v}\left(\mu_{l}\right)$ if and only if $\mu_{l^{s}} \subseteq \mathbb{F}_{q}\left(\mu_{l}\right)$.

(c) If $\mu_{l} \subseteq \bar{K}_{v}$ then $\mu_{l} \subseteq \mathbb{F}_{q}$.

(d) $\operatorname{Syl}_{p}\left(G_{\bar{K}_{v}}\right)$ is a non-trivial free pro-p group.

Proof. Fix an $l$-Sylow extension $\left(E_{l}, v_{l}\right)$ of $(K, v)$ relative to $K_{\text {sep }}$. Denote its residue field by $\bar{E}_{l}$. Then $G_{\bar{E}_{l}} \cong \operatorname{Syl}_{l}\left(G_{\bar{K}_{v}}\right)$. One has $\mu_{l} \subseteq E_{l}$ and $\mu_{l} \subseteq \bar{E}_{l}$. Also, the $l$-primary component of $\Gamma_{v_{l}} / \Gamma_{v}$ is trivial. Hence [E2, Lemma 2.4(b)] and Theorem 4.1(a) give $\left(\Gamma_{v_{l}}: l \Gamma_{v_{l}}\right)=\left(\Gamma_{v}: l \Gamma_{v}\right)=l$. Take $1 \leq s \leq \infty$ such that $\operatorname{Im}\left(\chi_{\bar{E}_{l}, l}\right)=1+l^{s} \mathbb{Z}_{l}$ (where we make the convention $l^{\infty}=0$ ). Then $G_{E_{l}} \cong \mathbb{Z}_{l} \rtimes G_{\bar{E}_{l}}$, where any $\sigma \in G_{\bar{E}_{l}}$ acts on the generator $\tau$ of $\mathbb{Z}_{l}$ according to $\sigma \tau \sigma^{-1}=\chi_{\bar{E}_{l}, l}(\sigma) \tau($ see $\S 1)$. It follows that $G_{E_{l}}(\mathrm{ab}) \cong\left(\mathbb{Z}_{l} / l^{s}\right) \times G_{\bar{E}_{l}}(\mathrm{ab})$.

The same analysis holds for $F$, so we obtain that $G_{F_{l}}(\mathrm{ab}) \cong\left(\mathbb{Z}_{l} / l^{s^{\prime}}\right) \times \mathbb{Z}_{l}$, where $F_{l}$ and $s^{\prime}$ are defined in a similar manner. Since the residue field $\bar{F}_{l}$ of $F_{l}$ is the $l$-Sylow extension of $\mathbb{F}_{q}$, it does not contain $\mu_{l}$. Hence $s^{\prime}<\infty$. If $s=\infty$ then we would obtain that $G_{\bar{E}_{l}}(\mathrm{ab}) \cong \mathbb{Z}_{l} / l^{s^{\prime}}$, which is impossible at positive characteristic. We conclude that $s=s^{\prime}<\infty$ and $G_{\bar{E}_{l}}(\mathrm{ab}) \cong \mathbb{Z}_{l}$. It follows that $\bar{E}_{l}, \bar{F}_{l}$ contain the same roots of unity of $l$-power order, and $G_{\bar{E}_{l}} \cong \mathbb{Z}_{l}$. As $\operatorname{cd}_{p}\left(G_{\bar{K}_{v}}\right) \leq 1$ [S, II-4, Prop. 3], (a) follows from Lemma 4.2.

To prove (b) it remains to observe that $\mu_{l^{s}} \subseteq \bar{K}_{v}\left(\mu_{l}\right)$ if and only if $\mu_{l^{s}} \subseteq \bar{E}_{l}$, and likewise for $\mathbb{F}_{q}$ and $\bar{F}_{l}$.

To prove (c) assume that $\mu_{l} \subseteq \bar{K}_{v}$ and $\mu_{l} \nsubseteq \mathbb{F}_{q}$. Then $G_{K}(l) \cong \mathbb{Z}_{l} \rtimes \mathbb{Z}_{l} ¥ \mathbb{Z}_{l}$ (§1). On the other hand, $G_{F}(l) \cong G_{\mathbb{F}_{q}}(l) \cong \mathbb{Z}_{l}[\mathrm{E} 2$, Lemma 2.1], a contradiction.

Finally, we prove (d). By $[\mathrm{S}, \mathrm{I}-37, \operatorname{Cor} .2], \operatorname{Syl}_{p}\left(G_{\bar{K}_{v}}\right)$ is indeed a free pro- $p$ group. Suppose that it is trivial. Then the maximal pro- $p$ Galois extension of $\mathbb{F}_{p}$ is contained in $\tilde{\mathbb{F}}_{p} \cap \bar{K}_{v}$. However, (b) and (c) imply that $\tilde{\mathbb{F}}_{p} \cap \bar{K}_{v} \subseteq \mathbb{F}_{q}$, a contradiction. 
Theorem 4.4. Let $K$ and $v$ be as in Theorem 4.1 and suppose that char $K=0$. Then:

(a) $\Gamma_{v}=p \Gamma_{v}$;

(b) $\bar{K}_{v}$ is perfect.

Proof. For any algebraic extension $E$ of $K\left(\mu_{p}\right)$ the $p$-torsion part of $\operatorname{Br}(E)$ is isomorphic to $H^{2}\left(G_{E}, \mathbb{Z} / p\right)=H^{2}\left(G_{E^{\prime}}, \mathbb{Z} / p\right)=0$ ([S, II-4, Prop. 3]; here $E^{\prime}$ is as before the extension of $F$ corresponding to $E$ with respect to a fixed isomorphism $\left.\sigma: G_{K} \rightarrow G_{F}\right)$. It follows that for every Galois extension $M$ of $E$ of degree $p$, the norm homomorphism $N_{M / E}: M^{\times} \rightarrow E^{\times}$is surjective (see e.g. [M, Th. 15.7]).

To prove (a), let $E=K\left(\mu_{p}\right)$ and let $u$ be the unique extension of $v$ to $E$. By Proposition $4.3, \bar{K}_{v}$ contains only finitely many roots of unity. Hence so does its finite extension $\bar{E}_{u}$. It follows that $\operatorname{Gal}\left(\tilde{\mathbb{F}}_{p} \bar{E}_{u} / \bar{E}_{u}\right) \cong \hat{\mathbb{Z}}$. Therefore there is an unramified extension $(L, w)$ of $(E, u)$ of degree $p$; thus $\Gamma_{w}=\Gamma_{u}$. By [E1, Lemma 1.2] and by Lemma 2.5 (for the extension $L / E),\left(\Gamma_{v}: p \Gamma_{v}\right)=\left(\Gamma_{u}: p \Gamma_{u}\right)=1$, as required.

To prove (b), let $T_{v}, V_{v}$ be again the inertia and ramification groups, respectively, of $v$ in $G_{K}$. By Proposition 3.3, $T_{v} \cap \sigma^{-1}(V) \neq 1$. From Theorem 4.1(b) we get $p \nmid\left(T_{v}: V_{v}\right)$. Since $V$ is pro- $p$, these two facts imply that the pro- $p$ group $V_{v}$ is non-trivial. Therefore we can take a tower of finite extensions $K\left(\mu_{p}\right) \subseteq E \subset M$ such that $M / E$ is a wildly ramified extension of degree $p$. Then the residue field extension $\bar{M} / \bar{E}$ is trivial. The surjectivity of $N_{M / E}: M^{\times} \rightarrow E^{\times}$ established above implies that $\bar{E}=\bar{M}^{p}=\bar{E}^{p}$; i.e., $\bar{E}$ is perfect. Hence so is $\bar{K}_{v}$.

\section{Constructions}

We conclude by showing that various restrictions made in our main results in $\S 4$ are indeed necessary.

Example 5.1. For every positive integer $r$ we construct a Henselian valued field $\left(K_{r}, u_{r}\right)$ of characteristic $p$ such that $G_{K_{r}} \cong G_{F}$ and $\Gamma_{u_{r}} / p \cong(\mathbb{Z} / p)^{r}$.

We first construct inductively countable Henselian discretely valued fields $\left(K_{r}, v_{r}\right)$ as follows: Let $\left(K_{1}, v_{1}\right)$ be a Henselization of $\mathbb{F}_{q}\left(t_{1}\right)$ with respect to the discrete valuation with uniformizer $t_{1}$. Assuming that $\left(K_{r}, v_{r}\right)$ has already been defined, let $L_{r}$ be a maximal totally tamely ramified extension of it. Then the (supernatural) degree $\left[L_{r}: K_{r}\right]$ is prime to $p$. Let $\left(K_{r+1}, v_{r+1}\right)$ be a Henselization of $L_{r}\left(t_{r+1}\right)$ with respect to its discrete valuation with uniformizer $t_{r+1}$. Since both $L_{r}$ and $K_{r}$ are countable, Proposition 1.3 implies that $G_{K_{r+1}} \cong G_{K_{r}}$.

Next we construct the valuations $u_{r}$ on $K_{r}$ inductively as follows: Take $u_{1}=$ $v_{1}$. Assuming that $u_{r}$ has already been defined, let $w_{r}$ be its unique prolongation to $L_{r}$. Let $u_{r+1}$ be the refinement of $v_{r+1}$ such that the residue valuation $u_{r+1} / v_{r+1}$ on $L_{r}$ is $w_{r}[\mathrm{R}]$. Since both $w_{r}$ and $v_{r+1}$ are Henselian, so is $u_{r+1}[\mathrm{R}$, pp. 210-211]. One has an exact sequence

$$
0 \rightarrow \Gamma_{w_{r}} \rightarrow \Gamma_{u_{r+1}} \rightarrow \Gamma_{v_{r+1}} \rightarrow 0
$$


of ordered abelian groups, and $\Gamma_{w_{r}}$ is convex in $\Gamma_{u_{r+1}}$. We obtain an exact sequence of abelian groups

$$
0 \rightarrow \Gamma_{w_{r}} / p \rightarrow \Gamma_{u_{r+1}} / p \rightarrow \Gamma_{v_{r+1}} / p \rightarrow 0
$$

Since the $p$-primary part of $\Gamma_{w_{r}} / \Gamma_{u_{r}}$ is trivial, $\Gamma_{w_{r}} / p \cong \Gamma_{u_{r}} / p \cong(\mathbb{Z} / p)^{r}[\mathrm{E} 2$, Lemma 2.4(b)]. Combining this with $\Gamma_{v_{r+1}} / p \cong \mathbb{Z} / p$, we conclude that $\Gamma_{u_{r+1}} / p \cong(\mathbb{Z} / p)^{r+1}$, as desired.

In fact, $K_{r}, L_{r}$ embed in a maximal totally tamely ramified extension $\left(M_{r}, w_{r}\right)$ of the $r$-dimensional local field $\mathbb{F}_{q}\left(\left(t_{1}\right)\right) \cdots\left(\left(t_{r}\right)\right)$ with its canonical discrete valuation of rank $r$ (see [FV, Appendix B]). By considering the restrictions of $w_{r}$ to these fields one can obtain an alternative proof that $\Gamma_{u_{r}} / p \cong(\mathbb{Z} / p)^{r}$.

Example 5.2. There exists a Henselian discretely valued field $(K, v)$ of characteristic $p$ such that $G_{K} \cong G_{F}, \bar{K}_{v}$ is imperfect, and $G_{\bar{K}_{v}} ¥ \hat{\mathbb{Z}}$. Indeed, take $(K, v)=\left(K_{2}, v_{2}\right)$ (with terminology as in Example 5.1). Then $\bar{K}_{v}=L_{1}$. Since $K_{1}$ is imperfect, so is its separable extension $L_{1}$. According to $\S 1, G_{L_{1}} \cong$ $F_{p}\left(\hat{\mu} \times G_{\mathbb{F}_{q}} ; \aleph_{0}\right) \times G_{\mathbb{F}_{q}}$. In particular, $\operatorname{Syl}_{p}\left(G_{L_{1}}\right)$ has infinite rank. Conclude that $G_{\bar{K}_{v}}=G_{L_{1}} \nRightarrow \hat{\mathbb{Z}}$.

Example 5.3. Let $(K, v)$ be a complete discretely valued field. Suppose that char $\bar{K}_{v}=p,\left|\bar{K}_{v}\right| \leq \aleph_{0}, G_{\bar{K}_{v}} \cong \hat{\mathbb{Z}}$, and $\bar{K}_{v}$ has the same group of roots of unity as $\mathbb{F}_{q}$ (e.g., this happens when $K$ is a finite extension of $\mathbb{Q}_{p}$ with residue field $\mathbb{F}_{q}$ ). Let $L / K$ be an arithmetically profinite totally ramified extension (for the definitions see [Wi] or [FV, Ch. III, §5]). In particular, if $[L: K]=\prod_{l} l^{n(l)}$, then $n(p)=\infty$ and $\sum_{l \neq p} n(l)<\infty$. The theory of fields of norms of FontaineWintenberger [Wi, 3.2.3] implies that $G_{L} \cong G_{\bar{K}_{v}((X))}$. By Corollary 1.2, the latter group is isomorphic to $G_{F}$. If $u$ is the extension of $v$ to $L$, then $\Gamma_{u}=p \Gamma_{u}$ and $\Gamma_{u} / l \cong \mathbb{Z} / l$ for $l \neq p$ prime.

Remark 5.4. Let $M$ be an $n$-dimensional local field such that its canonical valuation of rank $n$ has residue characteristic $p$ (cf. [FV, Appendix B]). From the discussion in $\S 1$ it follows that for every prime number $l \neq p$ one has

$$
G_{M}(l) \cong\left\langle\sigma, \tau_{1}, \ldots, \tau_{n} \mid \sigma \tau_{i} \sigma^{-1}=\tau_{i}^{q}, \tau_{i} \tau_{j}=\tau_{j} \tau_{i}\right\rangle_{\mathrm{pro}-l}
$$

Now let $K$ be a field such that $G_{K} \cong G_{M}$. Similarly to the proof of Theorem 4.1 one can show that there is a Henselian valuation $v$ on $K$ such that $\left(\Gamma_{v}: l \Gamma_{v}\right)=l^{n}$ for all primes $l \neq p$ and such that $\operatorname{char} \bar{K}_{v}=p$ or 0 .

\section{Acknowledgement}

This research was partly carried out during the first author's visit in the University of Nottingham in July 1998. He thanks the university for its hospitality. 


\section{References}

[AS] E. Artin and O. Schreier, Eine Kennzeichnung der reell abgeschlossenen Körper, Abh. Math. Sem. Univ. Hamburg 5 (1927), 225-231.

[AEJ] J.K. Arason, R. Elman and B. Jacob, Rigid elements, valuations, and realization of Witt rings, J. Algebra 110 (1987), 449-467.

[B] E. Becker, Euklidische Körper und euklidische Hüllen von Körpern, J. Reine Angew. Math. 268-269 (1974), 41-52.

[E1] I. Efrat, A Galois-theoretic characterization of p-adically closed fields, Isr. J. Math. 91 (1995), 273-284.

[E2] Pro-p Galois groups of algebraic extensions of $\mathbb{Q}$, J. Number Theory 64 (1997), 84-99.

[E3] - Construction of valuations from K-theory, Math. Res. Lett., to appear.

[Ed] O. Endler, Valuation Theory, Springer-Verlag, New York-Heidelberg, 1972.

[EE] O. Endler and A.J. Engler, Fields with Henselian valuation rings, Math. Z. 152 (1977), 191-193.

[Eg] A. Engler, Fields with two incomparable Henselian valuation rings, Manuscripta Math. 23 (1978), 373-385.

[FV] I. Fesenko and S. Vostokov, Local fields and their extensions: A Constructive Approach, Translations of Mathematical Monographs, 121, American Mathematical Society, Providence, RI, 1993.

[FJ] M. Fried and M. Jarden, Field Arithmetic, Ergebnisse der Mathematik und ihrer Grenzgebiete (3) [Results in Mathematics and Related Areas (3)], 11, Springer-Verlag, Berlin-New York, 1986.

[HJ] Y.S. Hwang and B. Jacob, Brauer group analogues of results relating the Witt ring to valuations and Galois theory, Canad. J. Math. 47 (1995), 527-543.

[K1] H. Koch, Über die Galoissche Gruppe der algebraischen Abschliessung eines Potenzreihenkörpers mit endlichem konstantenkörper, Math. Nachr. 35 (1967), 323-327. (German)

[K2] _ Galoissche Theorie der p-Erweiterungen, VEB Deutscher Verlag der Wissenschaften, Berlin, 1970.

[Kn] J. Koenigsmann, From p-rigid elements to valuations (with a Galois-characterisation of $p$-adic fields), with an Appendix by F. Pop, J. Reine Angew. Math. 465 (1995), 165-182.

[KPR] F.-V. Kuhlmann, M. Pank, and P. Roquette, Immediate and purely wild extensions of valued fields, Manuscripta Math. 55 (1986), 39-67.

[MSh] O.V. Mel'nikov and A.A. Sharomet, The Galois group of a multidimensional local field of positive characteristic, Matem. Sb. 180 (1989), 1132-1147 (Russian); English translation in Math. USSR-Sb. 67 (1990), 595-610.

[MSu] A.S. Merkur'ev and A.A. Suslin, K-cohomology of Brauer-Severi varieties and the norm residue homomorphism, Izv. Akad. Nauk USSR. Ser. Mat. 46 (1982), 1011-1046 (Russian); English translation in Math. USSR Izv. 21 (1983), 307-340.

[M] J. Milnor, Introduction to algebraic K-theory, Annals of Mathematics Studies, No. 72, Princeton University Press, Princeton, N.J, University of Tokyo Press, Tokyo, 1971.

[N1] J. Neukirch, Zur Verzweigungstheorie der allgemeinen Krullschen Bewertungen, Abh. Math. Sem. Univ. Hamburg 32 (1968), 207-215.

[N2] _ Kennzeichnung der p-adischen und endlichen algebraischen Zahlkörper, Invent. Math. 6 (1969), 269-314.

[P1] F. Pop, Galoissche Kennzeichnung p-adisch abgeschlossener Körper, J. Reine Angew. Math. 392 (1988), 145-175.

[P2] F. Pop, On Grothendieck's conjecture of birational anabelian geometry, Ann. Math. 139 (1994), 145-182. 
[PR] A. Prestel and P. Roquette, Formally p-adic fields, Lect. Notes Math., 1050, SpringerVerlag, Berlin-New York, 1984.

[R] P. Ribenboim, Théorie des Valuations, Séminaire de Mathématiques Supérieures, No. 9 (Été, 1964), Les presses de l'Université de Montréal, Montréal, 1968.

[S] J.-P. Serre, Cohomologie Galoisienne, Lecture Notes in Mathematics, No. 5, SpringerVerlag, Berlin-New York, 1965.

[Wi] J.-P. Wintenberger, Le corps des normes de certaines extensions infinies des corps locaux, applications, Ann. Sci. École Norm. Sup. (4) 16 (1983), 59-89.

[Wr] R. Ware, Valuation rings and rigid elements in fields, Canad. J. Math. 33 (1981), $1338-1355$.

Department of Mathematics and Computer Science, Ben Gurion University of the Negev, P.O. Box 653, BE'ER-Sheva 84105, ISRAEL

E-mail address: efrat@math.bgu.ac.il

Department of Mathematics, University of Nottingham, NG7 2RD Nottingham, ENGLAND

E-mail address: I.Fesenko@maths.nott.ac.uk 